\title{
Towards CPS Based Aircraft MRO
}

\author{
Kai Mertins, Thomas Knothe, and Pavel Gocev \\ Fraunhofer IPK, Pascalstr. 8-9, 10587 Berlin, Germany \\ \{kai.mertins, thomas.knothe, pavel.gocev\}@ipk. fraunhofer.de
}

\begin{abstract}
Cyber Physical Systems (CPS) receiving more and more industrial reality. In this paper a concept for the application of CPS for Aircraft maintenance repair and overhaul, sketched with airline partners, will be given. Based on industrial needs and requirements as well as the current state of the art the concept is describing a direct assignment of MRO-tasks to mechanic, tools and spare parts in order improve aircraft availability. The planning will be supported through simulation based generation of contextual MRO-tasks.
\end{abstract}

Keywords: Cyber Physical Systems for MRO, modelling and simulation, MRO Planning and Control.

\section{Introduction}

Availability and reliability of aircrafts are essential factors in the global competition of the airlines. Beside the operative execution of Maintenance, Repair and Overhaul (MRO) the planning and control of the MRO processes impacts the aircraft availability too. This emerges directly from the configuration complexities of ITSystems, variety of tools and spare parts as well as numerous locations with their qualified specialists. At present the planning and control processes are carried out sequentially including unnecessary loops, redundancies and interfaces using different media and various structures from the MRO demand until the aircraft serviceable. The complexity is even higher due to the mixture of the planned and unforeseen MRO work. The improvement potential is estimated on 1 to $3 \%$ of availability increase yielding an amount of two-digit EUR Millions per mid-sized airline.

There is a necessity for a novel solution that will eliminate current problems emerging from the above described situation:

- Changes of flight plans or unforeseen technical problems extend the Aircraft on Ground (AOG) time due to unavailable MRO-Resources.

- $\quad$ MRO-Process landscape causes long through put time on operational and administrative level.

- Diagnosis systems do not consider the all necessary and available data for prediction of possible malfunctions and failures.

- Large C- and D-Checks are carried out as separate projects, but not broken into small work packages (Single Running Tasks) that can be executed during the daily checks of the aircrafts. 
- The response data and information from the MRO-Processes are not promptly used by the suppliers, either for design and development of components, nor for the modernisation and further improvement.

In order to answer on these challenges in this paper a solution is proposed based on deployment of Cyber Physical Systems (CPS) for context-sensitive planning, control and realisation of aircraft MRO-Processes. CPS is defined by Broy (1) as a narrow connection of embedded systems with the global digital networks with the goal of monitoring and control of physical occurrences using sensors and actuators as well as communication equipment. The goal of the below described solution concept is to reduce the complexity in the execution of MRO-Processes and to shorten administrative process parts. The aircrafts will carry CPS-Components that will be networked in one Platform and will be able to take decisions based on the particular situation. Therewith the aircrafts will issue an MRO-Order directly to the MROOperation under consideration of the available time, resources and spare parts. The solution will generate Single Running Task for MRO that will be released in a flexible way reducing the through put time and AOG time, increasing the availability of the aircrafts. The paper will give an overview of the challenges and will describe the concept for a solution as well as the necessary steps towards the solution realisation in this complex environment.

\section{Industrial Challenges, Requirements and Needs}

The improvement potential as mentioned in chapter 1 is currently limited by the complexity of planning and control of frequent as well as unscheduled MRO events for different stations in accordance to the individual work demand for each aircraft. Cost intensive spare parts, specific tools and equipment as well as special qualified personal are only available in selected stations. For unforeseen events expensive maintenance flights are required or deletions have to be taken. In order to prevent flight deletions reserve aircrafts have to been foreseen. As consequence the utilization of the entire fleet will be reducing. Unsatisfied customers and additional effort for aircraft operations are additional consequences.

TOP Level Business Requirements for Aircraft MRO-Provider:

- Realization of direct and consequently free of loop processes for maintenance planning and control. This would reduce the current process duration drastically.

- Improvement of reactivity of the MRO-provider regarding sudden required maintenance and repair activities. It has to be implemented that mechanicresources, spare parts, tools at locations will be provided in accordance to flight-plan, diagnostic data by optimal use of maintenance slots.

- Optimize the implementation of the concept of "Single Running Tasks". This means, that a small maintenance step of complex C- and D-Checks can be 
applied during regularly daily line checks and enable the reduction of the normally long duration of these C- and D-Checks. Currently this improvement cannot be applied, because of the complexity of planning and control procedures.

- Correct estimation of "out of order" cases and integration into the maintenance planning and control. Through this the number of sudden cases will be reduced, which cannot be planned in beforehand. The time of "Aircraft on Ground"' has to be reduced and the availability improved.

- For Aircraft and Aircraft Equipment Provider:

- A fast feedback of specific flight and maintenance data for improved support of aircraft modernisation, which is performed sometimes annually. This leads to enhanced agility and customer orientation.

- Direct data integration of maintenance related feedback from aircraft operations and maintenance into the IT-Systems for aircraft development and modernisation.

Similar requirements and needs are relevant in other industries like rail.

\section{State of the Art}

The demand on solution can be deducted from the viewpoint of the MRO-Service provider and from aircraft producers. Usually the process of MRO planning and control in air traffic is supported by specific IT-Applications as TRAX, or similar (2). These systems consider diagnosis data as well as operative aircraft information. Typical for these solutions is a redundancy of acquisition of data and information, data management. Moreover there are information gaps along the information process chain that impact the realization of the process in negative manner. Furthermore the long planning chain from MRO demand to the MRO operation by the mechanic requires too much time and implies a lot of inefficient loops (Fig. 1).

Related to the planning and control Sampigethaya (3) describes on a conceptual level the functionality of a direct communication between aircrafts and one Cyber Physical System (CPS). Thereby a focus is put on security of networks and communication infrastructures and a distributed planning and control as well as integration of CPS in the MRO-Processes is not mentioned at all.

The interface between the diagnosis and the predicted MRO is considered by Lee (4) already for more than 10 years but the suggested solution cannot answer on the challenges to cope with the specific dependencies within the MRO in the context of complex circumstances as for example the holistic air traffic systems.

The aircraft producer Boeing developed in the last years the so called "Boeing e-Enabled Solution" on a base of a reference architecture which should enable a strategic connection and integration of business processes, human resources, aircrafts, information and knowledge. The optimized and transformed processes through direct Ordering of Mechanic personnel and their response for the accomplished work are not foreseen with this solution and therewith there is no simplification of the MRO 
planning and control processes. For the feedback aim between MRO and the producer there are various additional systems which besides the partial data integration are dependent on manual efforts (4).
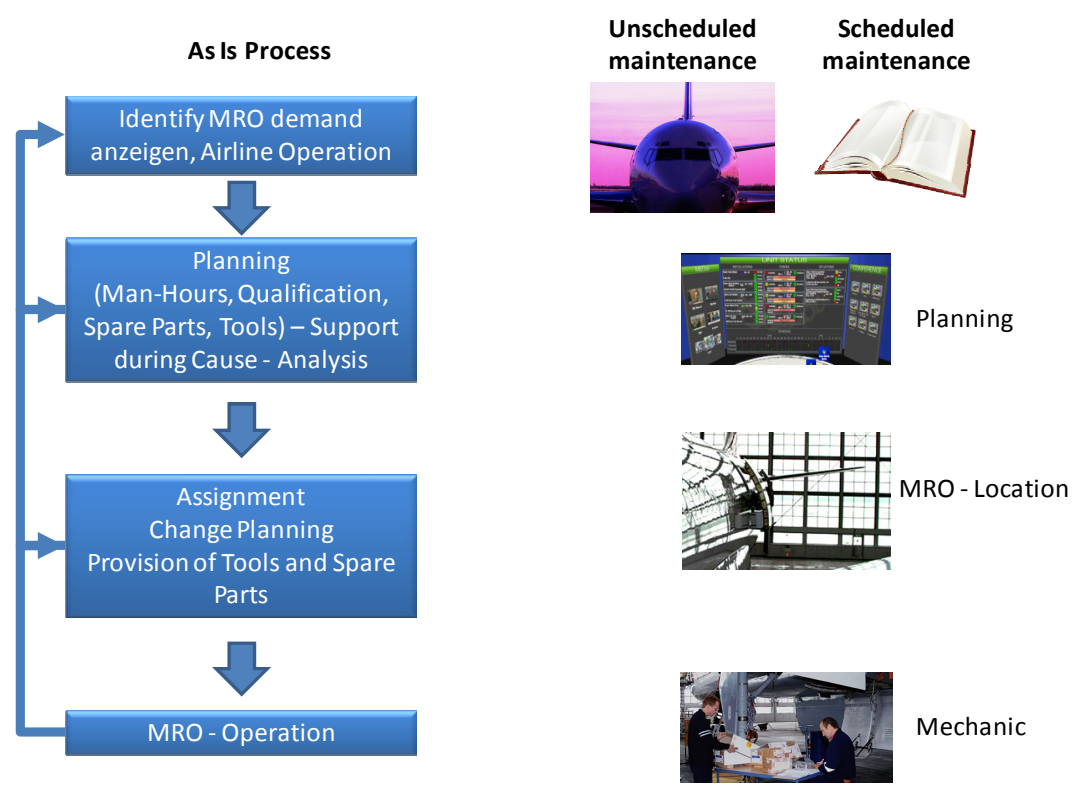

Fig. 1. AS-IS Process of Aircraft Maintenance Planning and Control

In the domain of services exist also reference models (6), like those which facilitate simulation of planning and control processes for MRO (7). The specific situation and the complexity of aircraft maintenance is only limited considered in the models. Methods for handling the complexity in integrated models are not inside these reference models. A suitable method would be a contextual integration of product and process models (8). The market is also supported by Integrated Product-Service Systems (IPS Systems) through contracting and material ordering up to response on carried out MRO work (9). In this case the MRO orders and responses need manual inputs of the MRO order progress status. IPS-Systems generate MRO-plans on the base of the available information and under deployment of rigid and predefined rules. These rules are usually operational charts, procedural conditions and are not flexible and not context sensitive. Routings are usually created manually and can be hardly adapted to the emerging situation. All these constrains and restrains the overall agility of the realization of MRO processes. The results from the research project SoPro (10; 11 ) is worth to contribute to the intelligent planning and control of MRO activities through self-organized production. In the SoPro project small computational unit eGrains have been developed which can be assembled on the MRO assets, transportation means, processing resources, tools and fixtures and that are equipped with agent based software and radio communication. Therewith through combination 
of negotiation of the agents the best optimal routing can be suggested and carried out, so a technical foundation for support of MRO processes is given. The German national project ARAMiS (12) is developing a concept for multiprocessors which offer new functionalities in the air traffic. Although there is no direct relation between to MRO, these processors can be utilized into CPS due to their efficiency and stability in order to enable direct assignment of the mechanical personnel by the system.

In general, the technological base for CPS extended aircraft avionic and MRO processes is available but there are numerous constraints which have to be adopted, integrated in the practical MRO environment. The mechanisms for system-integrated assignment, calculation, planning and control of MRO orders are still in an early phase and not applicable yet. The conditions for overall and comprehensive deployment of integrated MRO systems are still not available. The feedback from the MRO domain to the product design with the purpose of product improvement occurs at present only when corrective action and measures have to be taken or based on statistic evaluation. A direct context-sensitive feedback to the suppliers does not exist and therewith the improvement of processes and products are cost and effortintensive.

\section{Solution Concept}

The above described challenges were addressed by Fraunhofer IPK considering an aircraft MRO-process chain form aircraft component supplier, aircraft OEM up to airline and MRO service provider. The solution is related to a context-sensitive planning, control and realization of aircraft MRO-Processes based on deployment of Cyber Physical Systems (CPS) with the goal to reduce the complexity in the execution of MRO-Processes and to shorten the administrative part. The aircrafts and their components will carry CPS-Components that will be networked in one solution platform and will be able to take decisions based on the particular situation, and consequently will issue an MRO-Order directly to the MRO-Operation under consideration of the available time, resources and spare parts. The solution will generate Single Running Task for MRO that will be released in a flexible way reducing the through put time and Aircraft-on-Ground (AOG) time, increasing the availability of the aircrafts. The goal is to deploy and utilize the solution of Cyber-Physical Systems within the MRO-processes. Moreover the depending processes like product design, production planning and control, as well as ergonomics will be considered.

Due to the complexity of the whole concept, the solution will be developed on the basis of one or more use cases that will prepare the challenges and requirements for a solution from the industrial and business point of view. This will support the formal description of the necessary objects within the MRO domain, as well as their transformations along the MRO-Processes. In particularly the specifics of processes, resources, technologies, products and practices of MRO will be considered and their nature and features will be analyzed. The relations and dependencies between the objects (aircrafts, components and MRO-resources) within the MRO-processes will be described in one extended process model that will keep all relevant aspects and the respective interdependencies. 


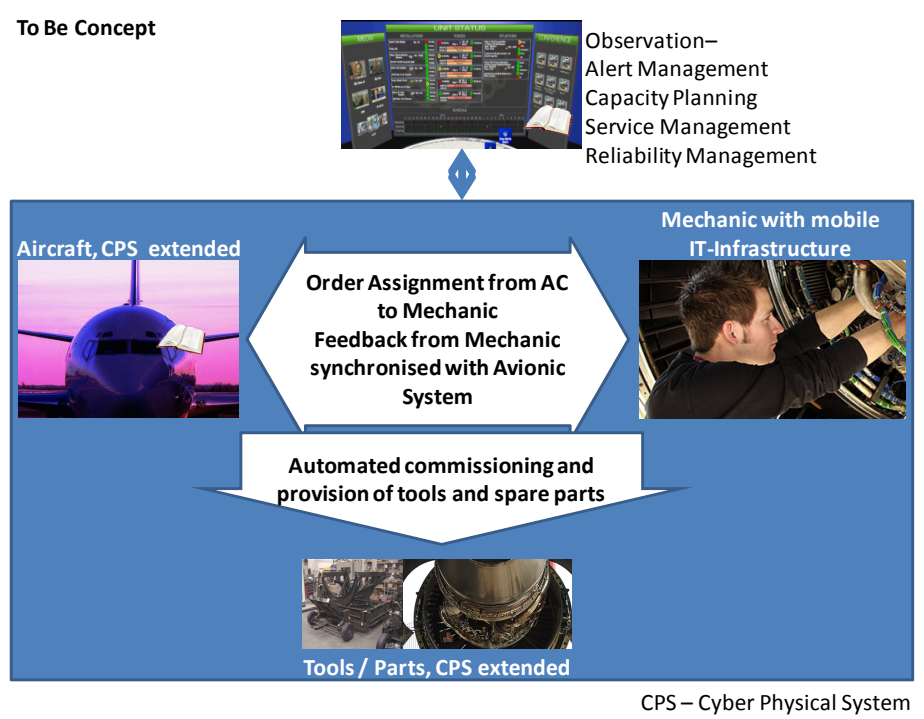

Fig. 2. TO-BE Concept of direct task assignment from Aircraft to mechanic, tools and parts

The solution will utilize the definition and description of the MRO-processes and activities that have to be performed to each aircraft component according to the defined plan or checks. This will include a list of activities to be carried out and necessary resources for each activity. The challenge is to cope with the real situation at the airlines. On one side the aircrafts are changing their geographical position with the time and on the other side the MRO-resources are spatially scattered in more locations. As not every MRO-location offers every MRO-service the solution will offer an execution of the MRO-processes (checks) in one optimal way. The aircrafts and their components that are a subject of MRO will be integrated into the MROplanning and control system in the way that they can be pro-active and react on the environment. This will be enabled through equipment the aircraft components with CPS-objects like sensors, actuators, RFID, respectively "small computers" that are able not only to read and write data and information but to process them and proactively to cooperate in one CPS based MRO system. In that way each aircraft component will carry with itself the information about the MRO-demand and necessary MRO-processes to be carried out. Moreover the aircraft component will be "aware" about the required MRO-resources that will be capable to perform the MROprocess. In regard to the current location of the aircraft and considering the flight plan each aircraft or component can match the MRO-process to be realized with the available resources at the same location. Matching the all criteria relevant for performing the MRO-process the aircraft or the component will be able to take a decision, to select a single running task to be realized, to generate a MRO-order for selected MRO-resource and to assign the MRO-work to the resources. All that will happen locally via direct communication between the aircraft or its component and the MRO-location and its resources. 
The personnel responsible for accomplishing the MRO-work will get the work instruction and all necessary information including the history values about the aircraft or component directly from the CPS-object carried by the aircraft or component. In a similar way the realized MRO-work and the findings as well as the performed changes and the information about the built spare parts can be sent to the CPS-object directly to the aircraft or component and therewith the history values will be updated in a very direct way of communication between the execution participant of the MRO-work and the aircraft or component that experienced the MRO-process.

The impact of the proposed solution can be multiple regarding the better performance of the MRO-Processes, improved utilization and sustainability of the systems, bringing together various information sources for better planning and control. Moreover this will foster the further development of the CPS-components and open the perspectives for new applications of CPS in other domains. One example is the railway sector with similar business conditions to synchronize overnight short maintenance, regular long lasting maintenance projects, timetable oriented operation and many maintenance locations as well as expensive equipment and parts. The new paradigm will drive the $\mathrm{R} \& \mathrm{D}$ community for new solutions that will improve the efficiency of the systems and will enable an accomplishment of complex challenges with the final goal of improvement of the economic well-being.

\section{Outlook and Further Steps}

Besides the opportunities of the described concept, the longterm aircraft product live cycle will be a barrier of the implementation of CPS based aircraft and CPS based aircraft maintenance. Nevertheless there are two midterm steps to go on the modernization way:

- Start with the implementation according to software updates for the aircraft avionic, because the infrastructure (e.g. wireless LAN on airports) and the technology (e.g. Boeing avionic) is currently available.

- Enhance the flight bag system (tablet PC for pilots, contain routes, maps and calculators, e.g. for fuel consumption estimation) with reading interface to aircraft data bus system. A communication system to the maintenance planning and control integrated on the flight bag system can support the direct processes from aircraft to maintenance staff. The improvement of the flight bag system is independent from aircraft design changes; the interface to the aircraft data bus already exists.

- Implement auto-id systems for expensive parts and tool as well as insure the data integration into maintenance planning and control system.

The solution approach affects a number of standards, especially aircraft architecture like ATA42 chapter „Aviation Industry Standards for Digital Information Security“, MRO-Standards, regarding MRO-operations from EASA, Part-145, Part-66, Part-M. In the near future these standards need to be checked and if necessary revised in order 
to allow CPS based aircraft maintenance. E.g. the part-145 is currently very much element oriented and on a status like ISO 9001 was in 1994. Here a process orientation is required, because the interaction of most of the elements of part-145 (e.g. staff qualification and repair procedure) have to be defined and implemented in their process context.

\section{References}

1. Broy, M.: Cyber-Physical Systems: Innovation durch softwareintensive eingebettete Systeme. In: Manfred Broy, C. (ed.). Springer, Acatech Diskutiert (2010)

2. TRAX, http://www.trax.aero/index_main.htm

3. Sampigethaya, E.A., Sampigethaya, K., Poovendran, R., Shetty, S., Davis, T., Royalty, C.: Future e-enabled aircraft communications and security: The next twenty years and beyond. In: Proceedings of the IEEE, Special issue on Aerospace Communications and Networking in the Next Two Decades (December 2011) (accepted)

4. Lee, E.: Intelligent Maintenance Systems: The Next Five Years and Beyond Transforming Condition-based Maintenance to Productivity and Service Innovation. Asset Management and Maintenance Journal, 6-13 (July 2010)

5. Aircraft Commerce. Integrating maintenance \& engineering IT systems with the OEMs. Aircraft Commerce (36), 25-34 (2004)

6. Fettke, P., Loos, P., Zwicker, J.: Business Process Reference Models: Survey and Classification. In: Kindler, E., Nüttgens, M. (Hrsg.) Business Process Reference Models. Proceedings of the Workshop on Business Process Reference Models (BPRM 2005), Satellite Workshop of the Third International Conference on Business Process Management (BPM), pp. 1-15 (2005)

7. Knothe, T.: Referenzmodell Auftragsdurchlauf für Dienstleistung und Verwaltung. In: Wenzel, S. (Hrsg.) Referenzmodelle für die Simulation in Produktion und Logistik, SCS Verlag, Delft (2000)

8. Girod, M., Schramm, A., Schultz, R., Wintrich, N.: Integrierte Produkt- und Prozessmodellierung. In: ZWF Zeitschrift für wirtschaftlichen Fabrikbetrieb, pp. 454-457 (June 2011)

9. MacInnes, R.L., Pearce, S.L.: Strategic MRO-A Roadmap for Transforming Assets into Competitive Advantages, pp. 253-278. Productivity Press (2003)

10. Hohwieler, E.: Die Fabrik der Zukunft steuert sich selbst / The factory of the future will steer itself. Industrial Insight, pp. 11-15 (2010)

11. Mertins, K., Rabe, M., Schallock, B.: Innovative production control based on decentralized intelligence. In: Marco, T., et al. (Hrsg.) Proceedings International Conference on Advanced Production Management Systems, Poliscript (2010)

12. ARAMIS,

http://www.kit.edu/downloads/pi/PI_2011_179_Neue_Computersys

teme_fuer_Auto_Bahn_und_Flugzeuge.pdf 